Article

\title{
On the Use of Statistical Entropy Analysis as Assessment Parameter for the Comparison of Lithium-Ion Battery Recycling Processes
}

\author{
Omar Velázquez-Martinez ${ }^{1}$ (1) Antti Porvali ${ }^{1}{ }^{(D}$, Karl Gerard van den Boogaart ${ }^{2}$, \\ Annukka Santasalo-Aarnio ${ }^{3}$, Mari Lundström ${ }^{1}$, Markus Reuter ${ }^{2}$ (D) and \\ Rodrigo Serna-Guerrero ${ }^{1, *(\mathbb{D})}$ \\ 1 Department of Chemical and Metallurgical Engineering, School of Chemical Engineering, Aalto University, \\ P.O. Box 16200, 00076 Aalto, Finland; omar.velazquezmartinez@aalto.fi (O.V.-M.); \\ antti.porvali@aalto.fi (A.P.); mari.lundstrom@aalto.fi (M.L.) \\ 2 Helmholtz Institute Freiberg for Resource Technology, Chemnitzer Str. 40, 09599 Freiberg, Germany; \\ boogaart@hzdr.de (K.G.v.d.B.); m.reuter@hzdr.de (M.R.) \\ 3 Department of Mechanical Engineering, School of Engineering, Aalto University, P.O. Box 14400, \\ 00076 Aalto, Finland; annukka.santasalo@aalto.fi \\ * Correspondence: rodrigo.serna@aalto.fi
}

Received: 31 January 2019; Accepted: 3 April 2019; Published: 23 April 2019

\begin{abstract}
The principle of the circular economy is to reintroduce end-of-life materials back into the economic cycle. While reintroduction processes, for example, recycling or refurbishing, undoubtedly support this objective, they inevitably present material losses or generation of undesired by-products. Balancing losses and recoveries into a single and logical assessment has now become a major concern. The present work broadens the use of relative statistical entropy and material flow analysis to assess the recycling processes of two lithium-ion batteries previously published in the literature. Process simulation software, that is, $\operatorname{HSC} \operatorname{Sim}{ }^{\circledR}$, was employed to evaluate with a high level of accuracy the performance of such recycling processes. Hereby, this methodology introduces an entropic association between the quality of final recoveries and the pre-processing stages, that is, shredding, grinding, and separation, by a parameter based on information theory. The results demonstrate that the pre-processing stages have a significant impact on the entropy value obtained at the final stages, reflecting the losses of materials into waste and side streams. In this manner, it is demonstrated how a pre-processing system capable of separating a wider number of components is advantageous, even when the final quality of refined products in two different processes is comparable. Additionally, it is possible to observe where the process becomes redundant, that is, where processing of material does not result in a significant concentration in order to take corrective actions on the process. The present work demonstrates how material flow analysis combined with statistical entropy can be used as a parameter upon which the performance of multiple recycling processes can be objectively compared from a material-centric perspective.
\end{abstract}

Keywords: material flow analysis; relative statistical entropy; circular economy; lithium-ion batteries; LIB recycling; process simulation

\section{Introduction}

The transition from a linear to a circular economy (CE) relies on the optimization of resource consumption (e.g., energy, raw materials) during the life-cycle of a product, that is, from extraction of virgin raw material, to production, manufacturing, consumption, end-of-life, and ultimately recycling [1]. However, product design dwindles the development of $C E$ due to the fact that complex 
technological products (e.g., batteries, mobile phones) are built with intrinsically linked components. Thus, recycling technologies need to find new processes to deal with the increasing complexity of consumer products. Parametrization, control, and optimization of resource efficiency in recycling processes consequently turn into key factors to achieve a CE.

Lithium-ion batteries (LIB) are one of the most ubiquitous products in today's economy [2], and its presence in the world is expected to increase steadily [3-7]. Consequently, the demand for raw materials to manufacture LIBs will increase. It is well known that exploitation of primary raw materials is becoming a more complicated activity, as the grade of mineral deposits suitable for extraction diminishes, along with the remote geographical location of extractive operations. As can be seen in Figure 1, a LIB presents a wide variety of elements and chemical compounds, which ought to be supplied in sufficient quantities to sustain the presence of LIB in the economy. Thus, efficient recycling processes of LIB will play an utterly important role in their future production.

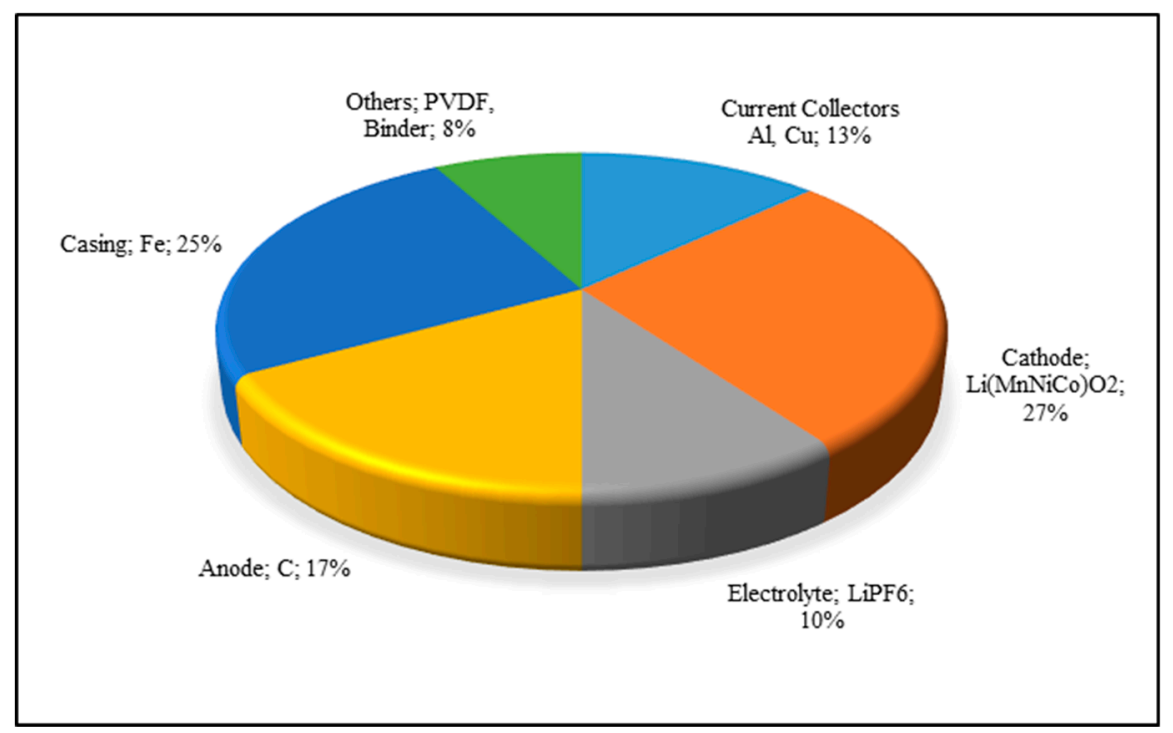

Figure 1. Components of a common Lithium-ion battery (LIB). PVDF stands for Polyvinylidene Fluoride [3-7].

Thus, understanding the true limits of recycling processes and effectively parametrizing their efficiency are key elements to optimize the reintroduction of LIB constituents into the economic cycle [8]. Some recently proposed recycling technologies claim to have achieved high efficiencies in the recovery of LIB components [9-11], and are some examples of the need for mechanical processing and upgrade to make the refining of a wider variety of materials feasible. Research on battery recycling technologies has advanced in the latter decades, from processes with a short number of stages and high energy consumption aimed only at the recovery of the metallic components [12] to extensive processes that recover almost $90 \%$ or all component, including metallic fractions of high commercial value $[5,9,10]$.

The current approach to measuring the efficiency of a recycling system is typically focused on the performance of a single, typically final, stage of the process and oversees the work conducted throughout the entire process, inevitably associated with material losses. However, the upgrade of material by mechanical processing is needed to produce high-quality feeds for the refining process $[13,14]$. Consequently, mechanical processes have an embedded impact in the final recovery that is ignored with benchmarks focused on a single transformation stage. Therefore, other tools to define efficiency from a more holistic perspective on recycling should be explored. Material Flow Analysis (MFA) coupled with Relative Statistical Entropy (RSE) present a suitable option to overcome the drawbacks of other traditional efficiency parameters. Using MFA-RSE, transformation processes can be analyzed from a systemic perspective, that is, accounting for the dilution or concentration of elements in all the streams of a system, including by-products and waste streams. Indeed, MFA offers a methodology for 
the systematic assessment of the flow of a substance throughout a system within a defined system boundary [15]. This approach generates a complete scope of the stock and flow of materials, which can be used to determine properties, such as RSE at a systemic level. In recent years, this methodology has been applied, for example, to identify and correct inefficiencies during Pb smelting operations [16] and as a baseline for $\mathrm{Cu}$ management in Europe [17]. Furthermore, in a recent publication by [18], MFA and RSE were employed to design and optimize a LIB recycling pre-processing system based on its entropy reduction capability. In this work, such methodology is used to determine the entropy evolution of PP stages and its implications on subsequent refining stages. A detailed description of the mathematical basis of the MFA-RSE method is provided in Section 2.2.

In an effort to turn the CE philosophy into a reality in the fields of metal processing, [19] presents a unique vision of a pyrometallurgical process. Therein, the authors make a comparison of two primary copper smelters employing simulation-based analysis. Such work evaluates the effect of circular economy practices, including analysis of mass losses, exergy and thermo-economics, and life cycle analysis. The work hereby presented acts in the same line of [19], providing a comparative study between two LIB recycling processes by MFA and RSE, supported by process simulation. Both recycling processes were designed exclusively differing on PP stages. Thus, the difference between RSE values describes the influence of different PP stages on the final entropy share of the recovered materials, uniquely giving an information weight to previous PP stages. It is expected that analysis with process simulation, MFA and RSE, in conjunction with analysis such as those presented by [19], provide parameters for an objective and systematic evaluation of processes to reach a true CE.

\section{Materials and Methods}

\subsection{LIB Recycling Processes}

The work hereby presented the analyses of two recycling processes using their ability to recover $\mathrm{Al}$ and cobalt oxalate for comparison (Figure 2). These LIB components were chosen as representative examples of LIB components potentially recovered via pyrometallurgical refining (represented in red in Figure 2) or hydrometallurgical processes (blue in Figure 2). Nevertheless, it is worth noting that the methodology hereby presented is not limited to these specific elements.

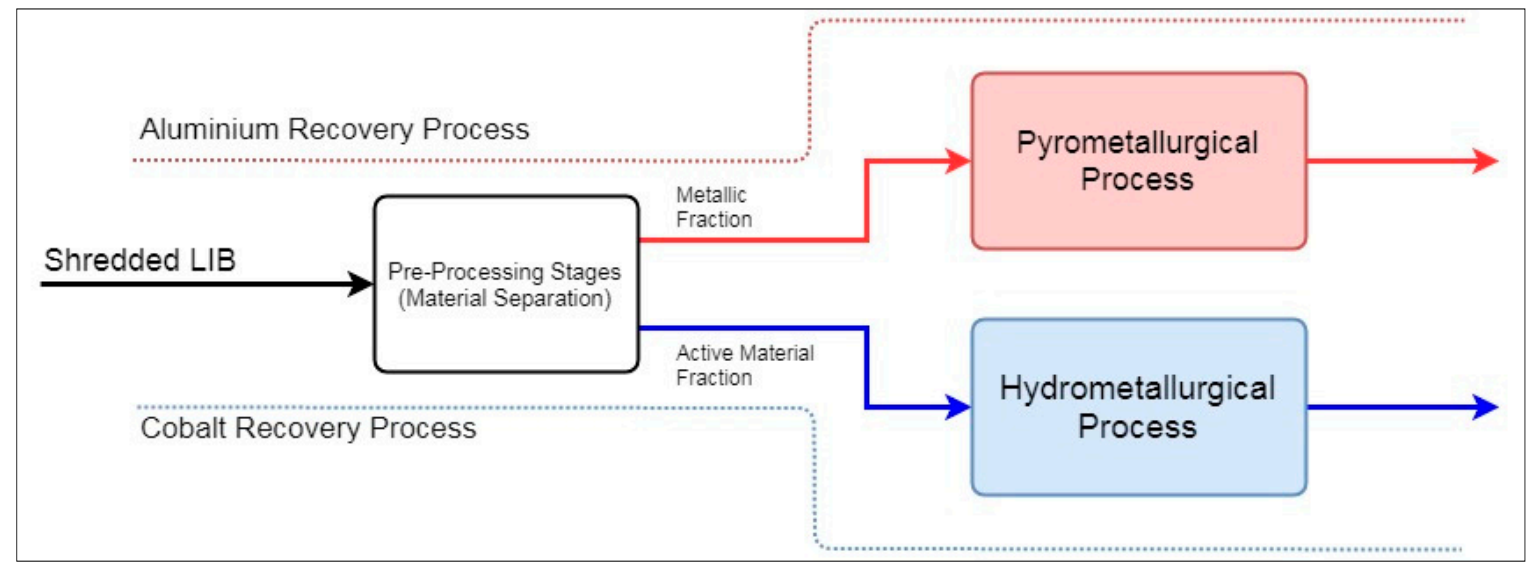

Figure 2. Flowsheet of the simulated recovery processes. LIB: Lithium-ion battery.

As seen in Figure 2, both pyro- and hydrometallurgical processes (described in detail in the following Section 2.1.2) are fed from the products of PP stages. Two different processes published in the literature were chosen for comparison and are hereby referred to as the "Aalto PP" [20] and the CSU PP $[9,21]$. These pre-concentration processes are described in Section 2.1.1. Therefore, the comparison would take place from the perspective of recycling processes for two different products: 
1. Aalto PP $+\mathrm{Al}$-recovery Pyrometallurgical Process vs. CSU PP + Al-recovery Pyrometallurgical Process

2. Aalto PP + Co-recovery Hydrometallurgical Process vs. CSU PP + Co-recovery Hydrometallurgical Process

Substituting the PP stages between the Aalto and CSU PP and keeping constant the hydro- and pyrometallurgical stages would depict the entropic influence of the PP on the final recoveries. HSC $\mathrm{Sim}^{\circledR}$ was utilized to simulate the processes and generate the corresponding mass balances to evaluate the values of statistical entropy.

\subsubsection{Simulation-Based Analysis of Pre-Processing Stages}

Aalto Pre-Processing

The Aalto PP is based on the process proposed by [20] based on the characterization and separation of real industrial LIB waste, and it is schematically described in Figure 3. This process is designed to obtain, with a relatively short number of steps, materials suitable for refining by hydro- and pyrometallurgical means.

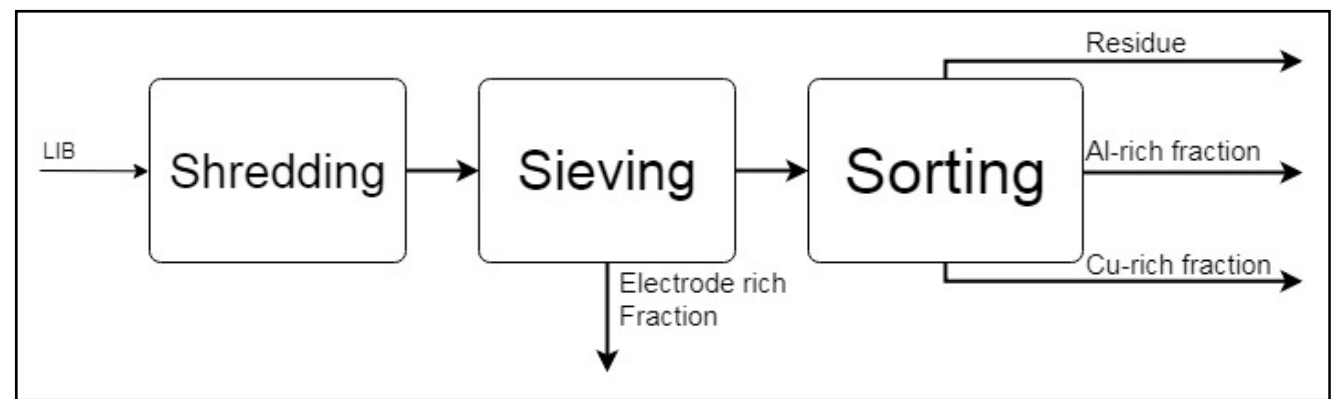

Figure 3. Flowsheet of the proposed process based on the results obtained by [20]. LIB: Lithium-ion battery.

According to the published data, the Aalto process produces an electrode-rich, Al-rich, $\mathrm{Cu}$-rich, and residue streams, employing three different process steps. The chemical composition of the streams obtained by the simulation in HSC Sim ${ }^{\circledR}$ of the Aalto PP using the reported values of feed composition presented in such work is stated in Table 1.

Table 1. Weight recoveries ( $\mathrm{wt} \%$ ) of the $\mathrm{Al}, \mathrm{Cu}$, electrode, and residues obtained by the Aalto pre-processing (PP) [20].

\begin{tabular}{ccccc}
\hline Element (i) & Al-Rich Fraction & Cu-Rich Fraction & Electrode Rich Fraction & Residue \\
\hline Al & $60.59 \%$ & $0.18 \%$ & $1.80 \%$ & $0.00 \%$ \\
C & $0.00 \%$ & $0.00 \%$ & $54.13 \%$ & $0.00 \%$ \\
Li & $1.20 \%$ & $0.45 \%$ & $3.78 \%$ & $2.02 \%$ \\
Co & $11.66 \%$ & $1.91 \%$ & $23.31 \%$ & $10.57 \%$ \\
Ni & $1.30 \%$ & $0.50 \%$ & $2.54 \%$ & $0.90 \%$ \\
Cu & $1.68 \%$ & $74.37 \%$ & $2.45 \%$ & $1.99 \%$ \\
Fe & $0.46 \%$ & $0.92 \%$ & $0.66 \%$ & $0.00 \%$ \\
Mn & $1.51 \%$ & $0.14 \%$ & $11.33 \%$ & $5.43 \%$ \\
Plastics & $21.59 \%$ & $21.53 \%$ & $0.00 \%$ & 79.09 \\
\hline
\end{tabular}

As observed in Table 1, even though this process was designed to obtain fractions of high purity, the obtained fractions after separation are still a mixture of elements. Hence, it is expected to observe separation inefficiencies from the RSE analysis. 
Central South University (CSU) LIB Mechanical Processing System

The second process studied hereby is the one proposed by $[9,21]$ (Figure 4 ). It was reportedly designed for the preparation of feed for hydrometallurgical treatment with the aim of recovering a wide variety of LIB components. Thus, it has a similar scope as the aforementioned Aalto process and is suitable for comparison.

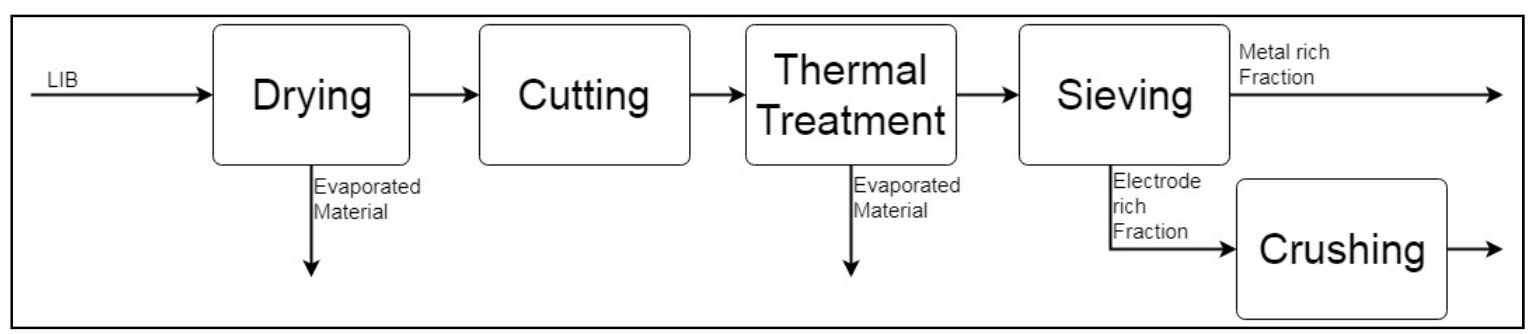

Figure 4. Flowsheet of the pre-processing stages for the recycling of Lithium-ion battery (LIB) described by $[9,21]$.

As the literature describes only the $w \mathrm{t} \%$ fed into the hydrometallurgical steps, the composition of shredded LIB fed into the PP steps was calculated using typical values of LIB products (Table 1) and adjusted to meet the quality requirements described by [9]. Table 2 presents the $\mathrm{wt} \%$ obtained by simulation for this system. Based on the process description, two streams of "evaporated material" are produced, consisting mainly of electrolyte and binders. As the methodology used in this work is based on the concentration of mass, it is vital to account for all possible source of losses for suitable analysis.

Table 2. Recovered wt $\%$ of the pre-processing steps reported in the literature $[9,21]$.

\begin{tabular}{ccc}
\hline Element (i) & Metal-Rich Fraction & Electrode-Rich Fraction \\
\hline $\mathbf{A l}$ & $34.25 \%$ & $0.09 \%$ \\
$\mathbf{C}$ & $1.18 \%$ & $31.10 \%$ \\
$\mathbf{L i}$ & $2.00 \%$ & $4.34 \%$ \\
$\mathbf{C o}$ & $1.39 \%$ & $36.88 \%$ \\
$\mathbf{N i}$ & $0.38 \%$ & $10.01 \%$ \\
$\mathbf{C u}$ & $54.80 \%$ & $0.15 \%$ \\
$\mathbf{M n}$ & $6.74 \%$ & $16.68 \%$ \\
$\mathbf{F e}$ & $0.87 \%$ & $0.23 \%$ \\
Others & $0.21 \%$ & $0.51 \%$ \\
\hline
\end{tabular}

It should be noted that the literature does not report the characteristics of the sieving system in the CSU process. Thus, the material sent to the pyrometallurgical process is assumed to be the totality of the metallic phase. As seen in Table 2, the metallic fraction is estimated to be heterogeneous, containing $\mathrm{Al}$ and $\mathrm{Cu}$ in significant quantities. This represents a significant difference with the Aalto PP, as its overflow of the sieving step is further treated to create concentrated streams of $\mathrm{Al}$ and $\mathrm{Cu}$.

\subsubsection{Simulation-Based Analysis of Pyro- and Hydrometallurgical Processes}

Simulation of the Al Recovery Process (Pyrometallurgy)

As a case study of high-temperature refining, a pyrometallurgical process for the recovery of $\mathrm{Al}$ was chosen, as described in Figure 5. The rotary kiln was simulated using the feed from either the "Al-rich" fraction from the Aalto PP or the "Metal-rich" fraction of the CSU PP (Tables 1 and 2, respectively).

The feed was heated to $800{ }^{\circ} \mathrm{C}$, powered by a methane burner $\left(\mathrm{CH}_{4}\right.$ stream in Figure 5) and a mixture of oxygen and nitrogen (Oxygen $+\mathrm{N}_{2}$ stream in Figure 5). Additionally, the kiln was fed with $\mathrm{KCl}$ and $\mathrm{NaCl}$ (Salt stream in Figure 5) to act as slag formers and remove impurities. At $800{ }^{\circ} \mathrm{C}$, organic material ( $\mathrm{C}$, binder) was lost as fumes or consumed as reduction agent; $\mathrm{Al}$ and $\mathrm{Cu}$ were sent to the 
molten phase (Molten Aluminium stream in Figure 5) [22], while other metals were processed as slag (Slag stream in Figure 5).

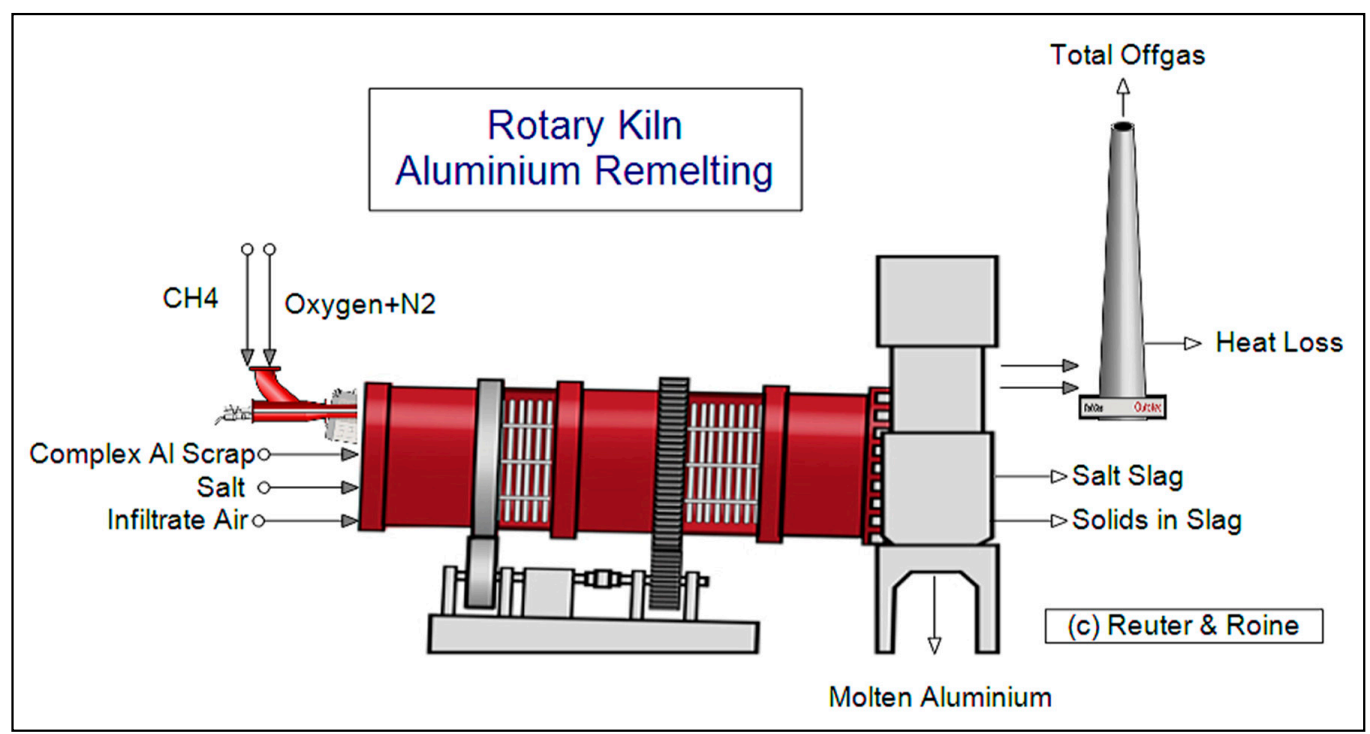

Figure 5. HSC Sim ${ }^{\circledR}$ simulation process diagram of the Al-recovery pyrometallurgical process.

Additionally, the kiln simulation was designed to emulate a feed of $\mathrm{Al}$ as foil shape particles with a thickness of $0.02 \mathrm{~mm}$, as it is a common size for this type of LIB current collectors [23]. The addition of this parameter increases the amount of alumina $\left(\mathrm{Al}_{2} \mathrm{O}_{3}\right)$ in the feed, as new surfaces of metallic $\mathrm{Al}$ (due to the shredding process) are exposed to ambient oxygen. Consequently, as the $\mathrm{Al}$-foil is shredded, the $\mathrm{Al}: \mathrm{Al}_{2} \mathrm{O}_{3}$ ratio increases. The different fusion points between $\mathrm{Al}_{2} \mathrm{O}_{3}$ and $\mathrm{Al}$, that is, $2072{ }^{\circ} \mathrm{C}$ and $660^{\circ} \mathrm{C}$, respectively, determine the amount of $\mathrm{Al}$ recovered by the kiln at the "molten Aluminium" stream (Figure 5). Thus, the presence of $\mathrm{Al}_{2} \mathrm{O}_{3}$ into the kiln reduces the recycling rate of metallic $\mathrm{Al}$, that is, $\mathrm{m}_{\mathrm{Al} \_ \text {feed }} / \mathrm{m}_{\mathrm{Al} @} @$ moltenAlstream. Table 3 presents the $\mathrm{Al}$ recycling rate and $\mathrm{Al}$ and $\mathrm{Cu} w \mathrm{w} \%$ at the "Molten Aluminium" stream obtained using streams from the Aalto and CSU PP stages.

Table 3. Al recovery results.

\begin{tabular}{ccc}
\hline Pre-Processing (PP) System & Al-wt $\%$ at “Molten Aluminium” & Cu-wt $\%$ at “Molten Aluminium” \\
\hline Aalto PP & $74.24 \%$ & $25.76 \%$ \\
CSU PP & $15.38 \%$ & $84.62 \%$ \\
\hline
\end{tabular}

The high variation in $\mathrm{Al}$ recycling rate between the two processes hereby studied (Table 3) can be attributed to two main reasons: (i) creation of $\mathrm{Al}_{2} \mathrm{O}_{3}$ during shredding and (ii) inclusion of $\mathrm{Cu}$ in the feeding material. As formerly mentioned, surfaces of pure $\mathrm{Al}$ obtained during shredding develop $\mathrm{Al}_{2} \mathrm{O}_{3}$. Hence, an ideal $\mathrm{Al}$ processing scenario would be where $\mathrm{Al}$ presents marginal amounts of its oxide form, for example, as Al-ingots, thus reducing the $\mathrm{Al}$ losses to slag. Furthermore, the presence of $\mathrm{Cu}$ in the stream decreases the concentration of $\mathrm{Al}$ in the molten stream. As observed in Tables 1 and 2, the metal-rich fraction obtained by the CSU PP contains a Cu concentration of $7.39 \%$, compared to $1.68 \%$ in the Aalto PP. The higher amount of $\mathrm{Cu}$ reported by the CSU PP, in addition to the Al losses as $\mathrm{Al}_{2} \mathrm{O}_{3}$, effectively turns the kiln into a $\mathrm{Cu}$ recovery process rather than an $\mathrm{Al}$ refining one. Even though the presence of $\mathrm{Cu}$ in the molten phase may be considered an impurity, the molten metallic phase could be potentially processed into a suitable Al-Cu alloy. However, such analysis is out of the scope of the current work. Thus, it is expected to have an RSE curve showcasing the dilution of Al during the final processing steps, represented by a higher entropy value. 
Simulation of the Cobalt Recovery Process (Hydrometallurgy)

As a working case for the analysis of hydrometallurgical processes, the recovery of cobalt oxalate $\left(\mathrm{CoC}_{2} \mathrm{O}_{4}\right)$, initially described by [9] and schematically presented in Figure 6, was chosen. From the perspective of a circular economy, this process is suitable for analysis as it claims that, under optimal conditions, it recovers most of the LIB elements, that is, $96 \%$ of $\mathrm{Li}, 95 \%$ of $\mathrm{Co}$, and ca. $99 \% \mathrm{Fe}, \mathrm{Mn}$, and $\mathrm{Cu}[9]$.

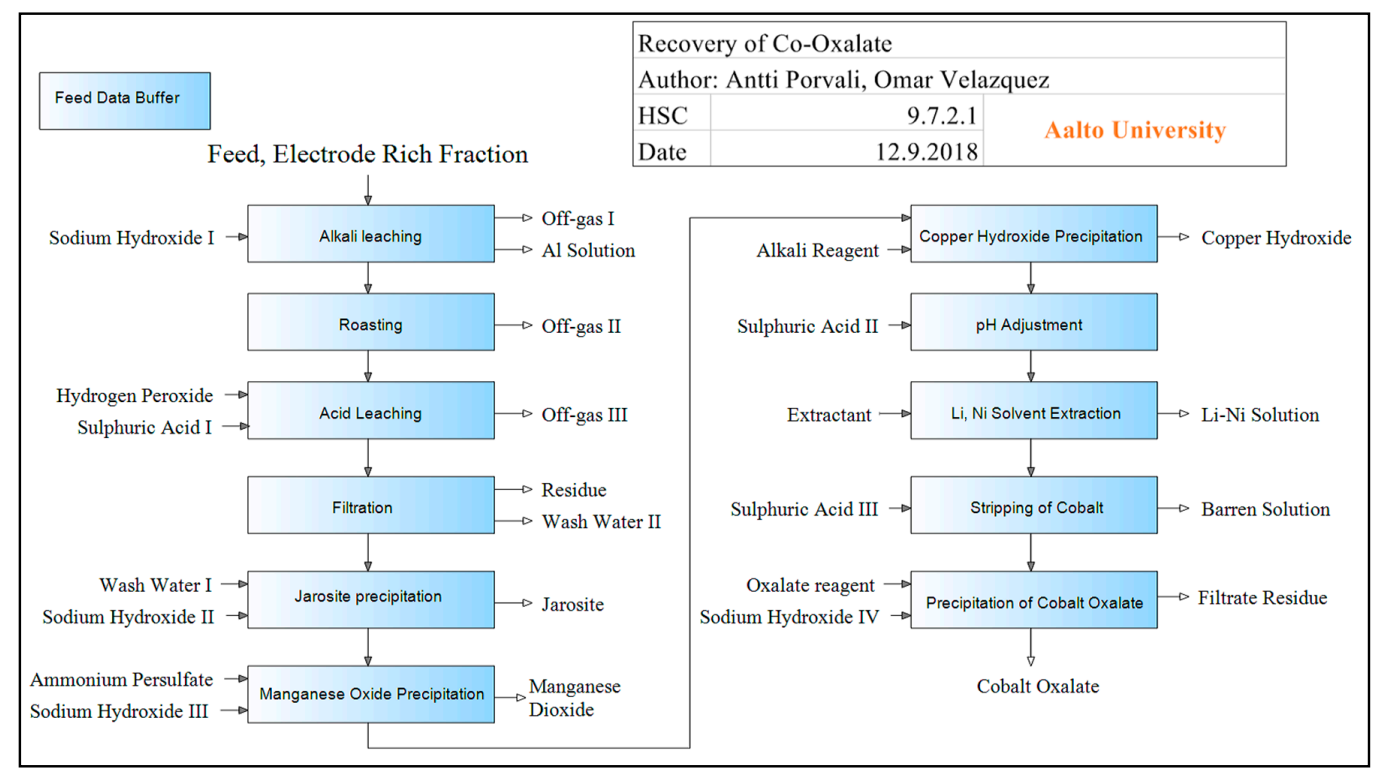

Figure 6. HSC $\operatorname{Sim}^{\circledR}$ simulation flowsheet of the hydrometallurgical process described by [9].

In a similar way, the hydrometallurgical process was simulated in HSC Sim ${ }^{\circledR}$ varying the feed material from the "electrode rich fractions" obtained by Aalto and CSU PP (Tables 1 and 2, respectively). Table 4 presents the recovery results per element according to the simulations of the hydrometallurgical process.

Table 4. The percentage recovery of elements of interest. Obtained from the simulation with HSC Sim ${ }^{\circledR}$ of the hydrometallurgical process.

\begin{tabular}{ccccccc}
\hline $\begin{array}{c}\text { Element } \\
\text { (i) }\end{array}$ & $\begin{array}{c}\text { Recovery } \\
\text { with Aalto } \\
\text { PP Stages }\end{array}$ & $\begin{array}{c}\text { Quality of } \\
\text { Element } \\
\text { (Aalto PP) }\end{array}$ & $\begin{array}{c}\text { Recovery } \\
\text { with CSU } \\
\text { PP Stages }\end{array}$ & $\begin{array}{c}\text { Quality of } \\
\text { Element } \\
\text { (CSU PP) }\end{array}$ & Extraction Stage & $\begin{array}{c}\text { Compound Form } \\
\text { of Element }\end{array}$ \\
\hline $\mathbf{A l}$ & $99 \%$ & $0.016 \%$ & $99 \%$ & $0,054 \%$ & Alkali Leaching & $\mathrm{Al}(\mathrm{OH})_{4}{ }^{-}$ \\
\hline $\mathbf{L i}$ & $93 \%$ & $0.13 \%$ & $92 \%$ & $0.18 \%$ & $\begin{array}{c}\mathrm{Li}-\mathrm{Ni} \text { Solvent } \\
\text { Extraction }\end{array}$ & $\mathrm{Li}^{+}$ \\
\hline $\mathbf{C o}$ & $89 \%$ & $40.1 \%$ & $89 \%$ & $40.1 \%$ & $\begin{array}{c}\text { Precipitation of } \\
\text { Cobalt Oxalate }\end{array}$ & $\mathrm{CoC}_{2} \mathrm{O}_{4}$ \\
\hline $\mathbf{N i}$ & $97 \%$ & $0.087 \%$ & $96 \%$ & $0.044 \%$ & $\begin{array}{c}\text { Li-Ni Solvent } \\
\text { Extraction }\end{array}$ & $\mathrm{Ni}^{+2}$ \\
\hline $\mathbf{C u}$ & $98 \%$ & $65.1 \%$ & $98 \%$ & $65.1 \%$ & $\begin{array}{c}\text { Copper Hydroxide } \\
\text { Precipitation }\end{array}$ & $\mathrm{Cu}^{2}(\mathrm{OH})_{2}$ \\
\hline $\mathbf{M n}$ & $98 \%$ & $62.7 \%$ & $97 \%$ & $62.7 \%$ & $\begin{array}{c}\text { Manganese Oxide } \\
\text { Precipitation }\end{array}$ & $\mathrm{MnO}_{2}$ \\
\hline $\mathbf{F e}$ & $99 \%$ & $34.6 \%$ & $99 \%$ & $34.6 \%$ & $\begin{array}{c}\text { Jarosite } \\
\text { Precipitation }\end{array}$ & $\mathrm{NaFe}_{3}(\mathrm{SO})_{4}(\mathrm{OH})_{6}$ \\
\hline
\end{tabular}

PP: pre-processing; CSU: Central South University. 
From Table 4, it is observed that results present a maximum $1 \%$ variation between both PP stages, including marginal differences compared to those described by [9]. Although the fate of graphite was not reported by [9], according to the simulation it is lost during the roasting step. Additional $\mathrm{C}$ required for the synthesis of cobalt oxalate $\left(\mathrm{CoC}_{2} \mathrm{O}_{4}\right)$ is added as the oxalate reagent $\left(\left(\mathrm{NH}_{4}\right)_{2} \mathrm{C}_{2} \mathrm{O}_{4}\right)$ during the last step of the process. Thus, the RSE value of $\mathrm{C}$ is expected to increase at the roasting stage and decrease during the precipitation of $\mathrm{CoC}_{2} \mathrm{O}_{4}$. As seen, the final recovery and purity of all products of the hydrometallurgical process are very similar and are independent of the PP stages. This justifies the need for a parameter that allows a deeper analysis and the subsequent comparison of processes beyond the quality of the final product.

\subsection{Mathematical Background of Material Flow Analysis and Relative Statistical Entropy}

This section briefly presents the mathematical background used in this work to describe the evolution of RSE based on the developments by [15]. Recently, our research group published a proof-of-concept on the use of RSE and MFA for the analysis of mechanical separations using a sieving stage as working example [18]. For the interested reader, a more detailed description of the MFA-RSE methodology can be found in such work.

Briefly, the statistical entropy $h_{i, s}$ of the $i t h$ component in the sth process stream is a function of its concentration and corresponding standardized mass fraction. In the context of statistical entropy, a component can represent any constituent of the stream with a defined concentration, which could be either a chemical compound or element. For the purposes of this work, the analysis is based on components at the elemental level, for example, $\mathrm{Al}$ and $\mathrm{Co}$. This parameter is measured in bits of information [bits], and it is obtained by employing Equation (1):

$$
h_{i, s}=-\widetilde{m}_{i, s} c_{i, s} \log _{2}\left(c_{i, s}\right) \geq 0
$$

where $c_{i, s}$ is the concentration [\%] and $\widetilde{m}_{i, s}$ is the standardized mass fraction [dimensionless] [15]. The latter is obtained by Equations (2) and (3):

$$
\begin{aligned}
\widetilde{m}_{i, s} & =\frac{\dot{m}_{s}}{\sum_{s} \dot{X}_{i}} \\
\dot{X}_{i} & =\dot{m}_{s} c_{i, s}
\end{aligned}
$$

where $\dot{m}_{S}$ is the total mass-flow [mass/time] in the sth process stream, and $\dot{X}_{i}$ is the total substance flow [mass/flow]. Thus, the statistical entropy value of the $i$ th component in the $q$ th stage $\left(H_{i, q}\right)$ is obtained by the sum of $h_{i, s}$ values in a stage, presented in Equation (4):

$$
H_{i, q}=\sum_{q} h_{i, s}
$$

The relative value of statistical entropy (RSE), Equation (6), makes possible the comparison between compounds in the system and between systems. RSE requires an arbitrary value of entropy to operate as a benchmark, whereas, Equations (5) serves this purpose.

$$
\begin{gathered}
H_{i, q}^{\text {input }}=\log _{2}\left[\sum_{q} \widetilde{m}_{i, q}\right] \\
R S E_{i, q}=\frac{H_{i, q}}{H_{i, q}^{\text {input }}}
\end{gathered}
$$

In this work, it is proposed to employ the entropy level of the feeding material. $H_{i, q}^{\text {input }}$ is set as the benchmark. This approach, thus, allows the objective comparison between both the analyzed processes. 
The analysis conducted by MFA and RSE can be graphically represented as a stage-node diagram, such as the example depicted in Figure 7.

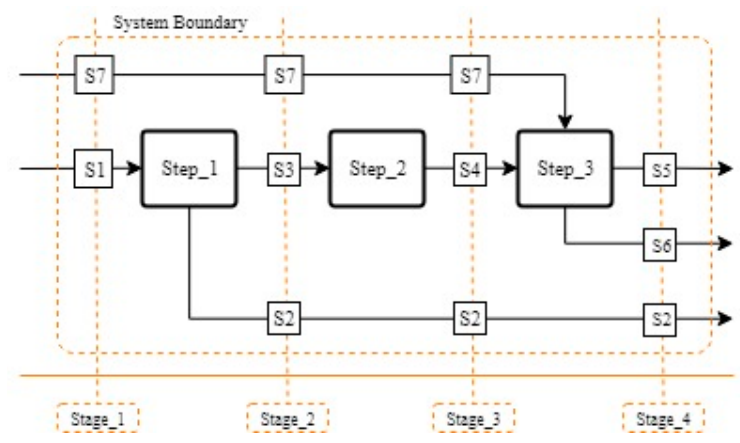

Figure 7. Stage-node diagram of a three-step system with two inputs and three outputs.

Figure 7 presents the stage-node diagram of a system with three processing steps, hence 4 stages, with one feeding input stream (S1), one input stream at ending step (S7), and three different output streams (S5, S6, and S2). The RSE value at any given stage is defined by the streams involved (i.e., produced, fed, or input) during the same and previous processes. As an example, Stage_2 in Figure 7 is defined by streams S2, S3, and S7, while the 4th Stage by S2, S5, and S6.

\subsection{Application of MFA and RSE to Recovery Processes}

This section presents the application of the MFA and RSE methodology on the recycling processes under study. The systemic approach is depicted in the form of a stage-node diagram. This approach is shown with Aalto PP and CSU PP in Figures 8 and 9, respectively. For reference, Table 5 describes the legend of unit operations, unit processes, and streams used in the stage-node diagrams.

Table 5. List of unit operations, processes, and streams used in node diagrams- Figures 10 and 11.

\begin{tabular}{lll}
\hline & Unit Operations & \\
\hline A. Drying & G. Roasting & M. $\mathrm{pH}$ Adjustment \\
B. Cutting/Shredding & H. Acid Leaching & N. SeX (Li, Ni) \\
C. Low Heat Thermal Treatment & I. Filtration & O. Stripping of Cobalt \\
D. Sieving & J. Jarosite Precipitation & P. $\mathrm{CoC}_{2} \mathrm{O}_{4}$ Precipitation \\
E. Crushing & $\mathrm{K} . \mathrm{MnO}_{2}$ Precipitation & Q. Sorting \\
F. Alkali Leaching & L. Cu(OH) $)_{2}$ Precipitation & R. Rotary Kiln \\
\hline & \multicolumn{1}{c}{ Stream List } & \\
\hline 0. Lithium-ion battery (LIB) & 1. Electrode Rich Fraction & 2. Residue \\
3. Al-rich Fraction & 4. Cu-rich Fraction & 5. Evaporated Material I \\
6. Evaporated Material II & 7. Metal-rich Fraction & 8. KCl \\
9. NaCl & 10. Slag & 11. Molten Aluminium \\
12. Sodium Hydroxide I & 13. Hydrogen Peroxide & 14. Sulphuric Acid I \\
15. Wash Water I & 16. Sodium Hydroxide II & 17. Ammonium Persulfate \\
18. Sodium Hydroxide III & 19. Alkali Reagent & 20. Sulphuric Acid II \\
21. Extractant & 22. Sulphuric Acid III & 23. Sodium Hydroxide IV \\
24. Oxalate Reagent & 25. Off-gas I & 26. Al Solution \\
27. Off-gas II & 28. Off-gas III & 29. Residue \\
30. Wash Water II & 31. Jarosite & 32. Manganese Oxide \\
33. Copper Hydroxide & 34. Li-Ni Solution & 35. Barren Solution \\
36. Filtrate Residue & 37. Cobalt Oxalate & \\
\hline
\end{tabular}

Figures 8 and 9 present the systemic approach of the RSE and MFA methodology, respectively, as the analysis is based on all the information in the streams (see Section 2.2) within the defined boundaries. In addition, the influence of an input stream on the previous stages and that of the output stream on subsequent stages is observed from the continuous solid lines entering and exiting the different processes. For instance, the input streams of oxalate reagent and sodium hydroxide (Streams 24) into 
the " $\mathrm{P}$ " process (Figures $8 \mathrm{a}$ and $9 \mathrm{a}$ ) have an influence on the entropy value from the initial stages of the system, that is, S1. Hence, their entropy share would influence each transformation stage from the initial stages until the end of the processes. Using other methods of analysis, this influence was most likely neglected. As observed in these diagrams, the recycling systems already depict differences. For instance, the recycling processes utilizing the CSU PP (Figure 9) present a slightly larger amount of stages compared to the system with Aalto PP stages (Figure 8).

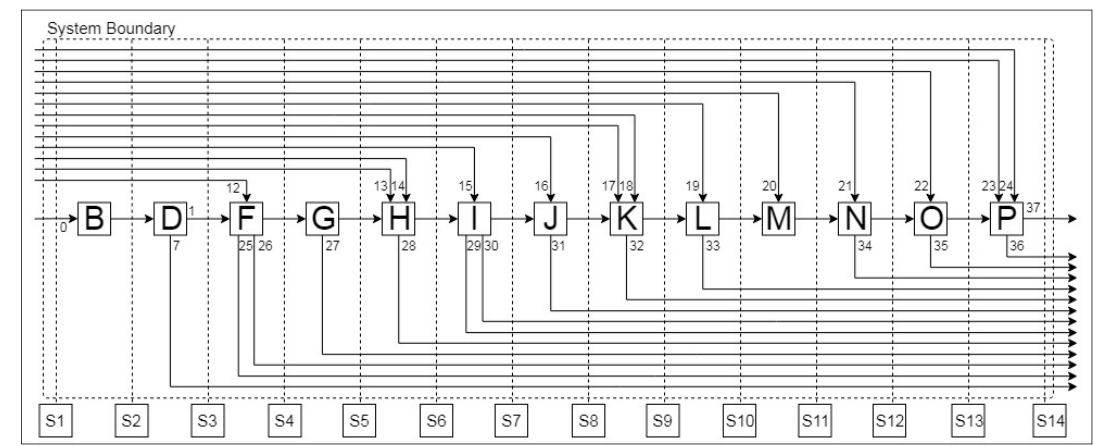

(a)

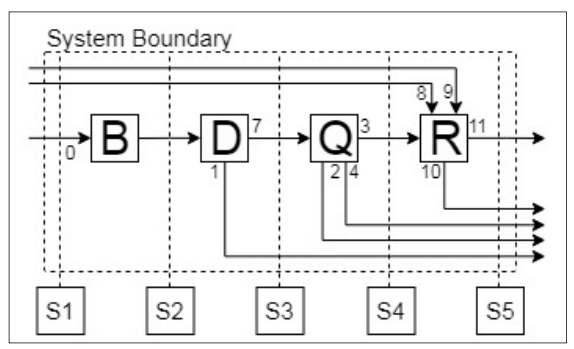

(b)

Figure 8. Electrode treatment (a) and Al-recovery (b) processes with Aalto pre-processing (PP) stages.

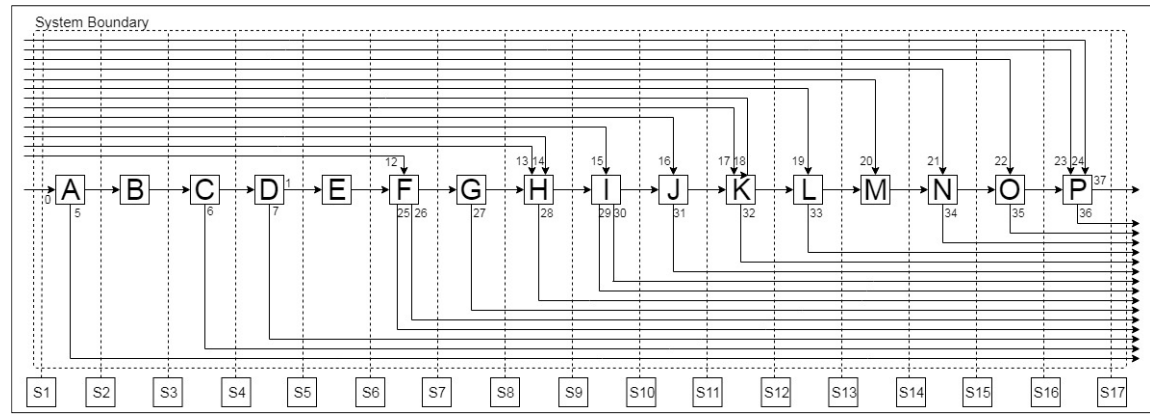

(a)

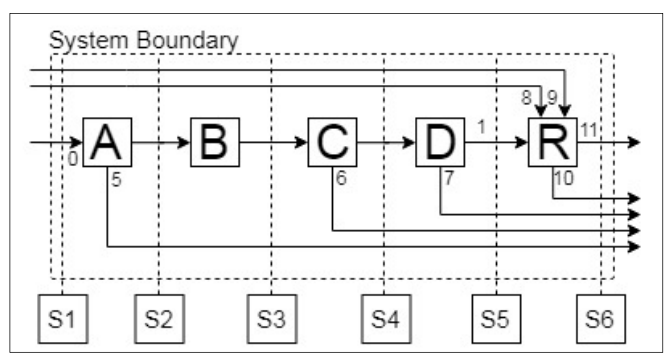

(b)

Figure 9. Electrode treatment (a) and Al-recovery (b) processes with pre-processing (PP) stages reported in the literature. 


\section{Results and Discussion}

The RSE Diagram, Figures 10 and 11, describe the evolution of the RSE of the recycling process described in Section 2.3. An RSE curve with negative slope reflects the concentration of the element, other possible behavior, that is, positive slope or plateau, and reflects a dilution or unchanged concentration, respectively.

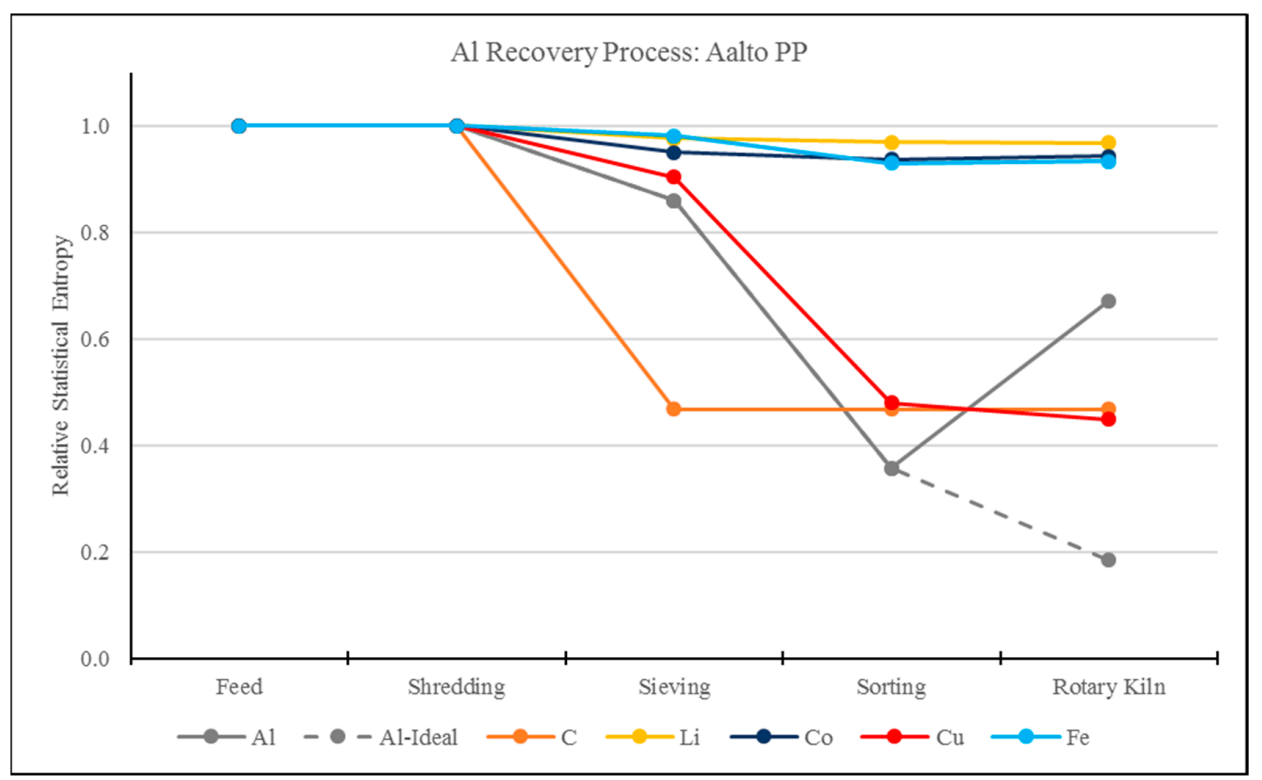

(a)

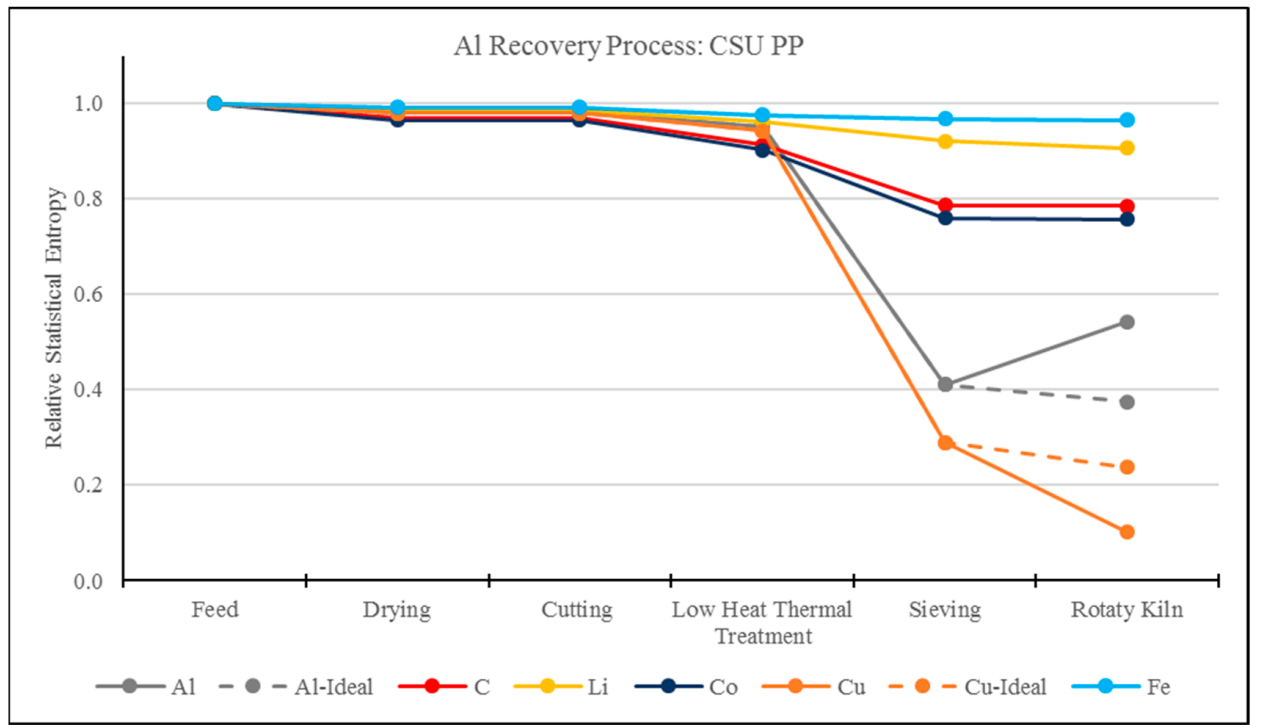

(b)

Figure 10. Al recycling Loops. (a) Aalto pre-processing (PP), (b) Central South University (CSU) PP.

\subsection{RSE Analysis of the Aluminum Recovery Processes}

Figure 10 presents the RSE Analysis of the Al-recovery process using Aalto PP (Top) and CSU PP (Figure 10b). Additionally, the process fed by the CSU PP, Figure 10b, keeps the material in a relative constant RSE value until the sieving step; thus, the initial four stages have a minor impact on concentration. In comparison, the Aalto PP presents an earlier decrease in entropy value, as all pre-concentration stages have the purpose of separating LIB components. In both cases, the sieving system produces a major entropy reduction. 
The latter stages of Figure 10 describe the entropy change due to the pyrometallurgical process. Observing the process employing Aalto PP stages (Figure 10a), it is determined that the Al curve (blue solid line, Figure 10a) increases due the lower amount of pure Al present in the molten phase rate. For comparison, the creation of $\mathrm{Al}_{2} \mathrm{O}_{3}$, as a consequence of shredding foil, directly affects the creation of entropy during the final stage. Hence, this system generates a higher amount of statistical entropy since new oxidized surfaces are created with the reduction of particle size as $\mathrm{Al}$ is diluted to $\mathrm{Al}_{2} \mathrm{O}_{3}$. The corresponding dashed line for $\mathrm{Al}$ represents an ideal scenario where $\mathrm{Al}$ ingots are produced with marginal levels of alumina $\left(\mathrm{Al}_{2} \mathrm{O}_{3}\right)$ that are lost into the slag phase. The process employing CSU PP stages (Figure 10b) presents the same trend of entropy increase of Al (gray solid line, Figure 10b), accompanied by a decrease in RSE value of $\mathrm{Cu}$ (solid line). Since $\mathrm{Al}$ is not sorted out from the metallic fraction in the CSU PP, its loss into the slag phase increases the $\mathrm{Cu}$ concentration in the product molten phase. The absence of a $\mathrm{Cu}-\mathrm{Al}$ separation stage, in this case, is effectively favoring the recovery of $\mathrm{Cu}$, instead of $\mathrm{Al}$, with the kiln.

A further implication of the $\mathrm{Cu}-\mathrm{Al}$ sorting stage in the Aalto process on the pyrometallurgical refining stage can be observed in the interdependency of the final RSE values of $\mathrm{Cu}$ and $\mathrm{Al}$. While in the Aalto process, the final values of $\mathrm{Cu}$ RSE are not significantly influenced by the presence or absence of $\mathrm{Al}_{2} \mathrm{O}_{3}$ dilution, in the $\mathrm{CSU}$ process, $\mathrm{Al}$ and $\mathrm{Cu}$ appear to be heavily linked. Indeed, in the latter case, the loss of $\mathrm{Al}$ in the form of $\mathrm{Al}_{2} \mathrm{O}_{3}$ (dilution) represents a concentration of $\mathrm{Cu}$, seen from the $\mathrm{Al}$ and $\mathrm{Cu}$ RSE curves trending in opposite directions (Figure 10b). The dashed lines of $\mathrm{Al}$ and $\mathrm{Cu}$ in Figure 10 represent the ideal scenario where the $\mathrm{Al}$ is processed with marginal levels of $\mathrm{Al}_{2} \mathrm{O}_{3}$. In the CSU process, the $\mathrm{Cu}$ final RSE value is comparatively higher as it is diluted with $\mathrm{Al}$ in the molten phase. On the other hand, the additional separation of $\mathrm{Al}$ in the metal-rich phase by the Aalto PP dissociates $\mathrm{Al}$ from $\mathrm{Cu}$ as seen from the horizontal $\mathrm{Cu}$ RSE value and positive slope Al RSE value (Figure 10a).

\subsection{RSE Analysis Electrode Treatment Processes}

Figure 11 depicts the RSE analyses for the hydrometallurgical processes, fed by Aalto and CSU PP stages, top and bottom, respectively. Similar to the pyrometallurgical process, both of the hydrometallurgical processes presented are fed with material containing the same elements but with slight differences in concentration (Tables 1 and 2). As observed in Table 4, the final recovery and quality in both cases are similar. Thus, the analysis of MFA and RSE is bound to provide more detailed information about the influence of PP stages on the final recyclates.

Initially, both processes present abrupt oscillations in RSE value after the PP stages due to the addition of the chemical reagents, hence, influencing the concentration of components in the streams. In general terms, hydrometallurgical processes are designed to separate, by chemical means, the elements by the addition of reagents to form more stable compounds, marginally affecting the rest of elements in ideal cases. Consequently, this may not represent a concentration of a certain element in some cases, as its extraction may be a result of dilution by a reagent. As an example, high purity $\mathrm{Al}$ foil [23] is diluted to aluminum hydroxide in the presence of sodium hydroxide. Consequently, the RSE curve of $\mathrm{Al}$ (light blue line in Figure 11) reflects the dilution of $\mathrm{Al}$ in the "Alkali Leaching" step. On the other hand, Fe is found in its diluted form, present in LIB either as cathode dopant [9] or as residues of steel casing (Table 1), and, by the effect of the chemical treatment, its relative concentration increases (Figure 11). Jarosite is a concentrated form of the Fe ions found in LIB; thus, the RSE curve for this element depicts a decrease in entropy towards the stage of "Jarosite Precipitation". In both cases, the extraction of jarosite and aluminum hydroxide before the last stage of the process would influence the subsequent stage entropy values. Nevertheless, the abrupt negative slope of the $\mathrm{Al}$ curve prior to "Alkali Leaching" stage is due to the physical separation carried out during the PP, whereas, the posterior increase in RSE value in the same curve is due to the dilution of $\mathrm{Al}$ to ions of $\mathrm{Al}(\mathrm{OH})$.

Co presents a similar behavior in both processes (dark blue solid line in Figure 11), initially undergoing a dilution step due to the addition of $\mathrm{NaOH}$ and an immediate concentration during roasting. The further dilution of $\mathrm{Co}$ is due to the addition of chemical reagents in subsequent steps for 
the extraction of all other elements, marginally affecting its entropy value. During the last steps of Co process, this element is once more diluted due to the addition of oxalate reagent and $\mathrm{NaOH}$ before the " $\mathrm{CoC}_{2} \mathrm{O}_{4}$ Precipitation" step where entropy decreases due to the formation of this compound, a concentrated form of $\mathrm{Co}$, and separation into a single stream.

The level of readiness, that is, whether a material is suitable for use in the form they were extracted, is affected by the dilution or concentration of the material. It is clear that this concept is subjective and depends on the intended application of the material. However, the forms of the extracted materials, that is, jarosite, manganese dioxide, copper hydroxide, Li-Ni Solution, and cobalt oxalate, may require further processing to become metallic raw materials or to be re-introduced as raw materials for LIB, which from the circular economy perspective is the ultimate objective.

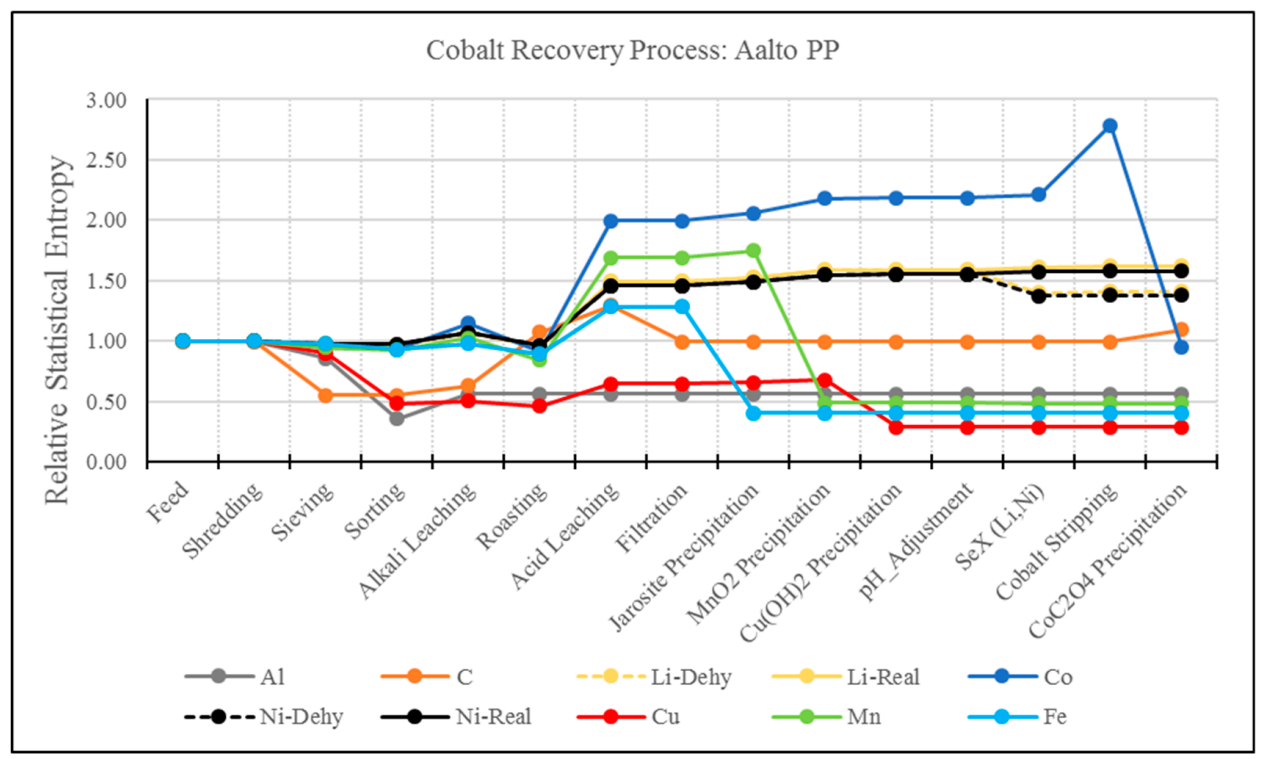

(a)

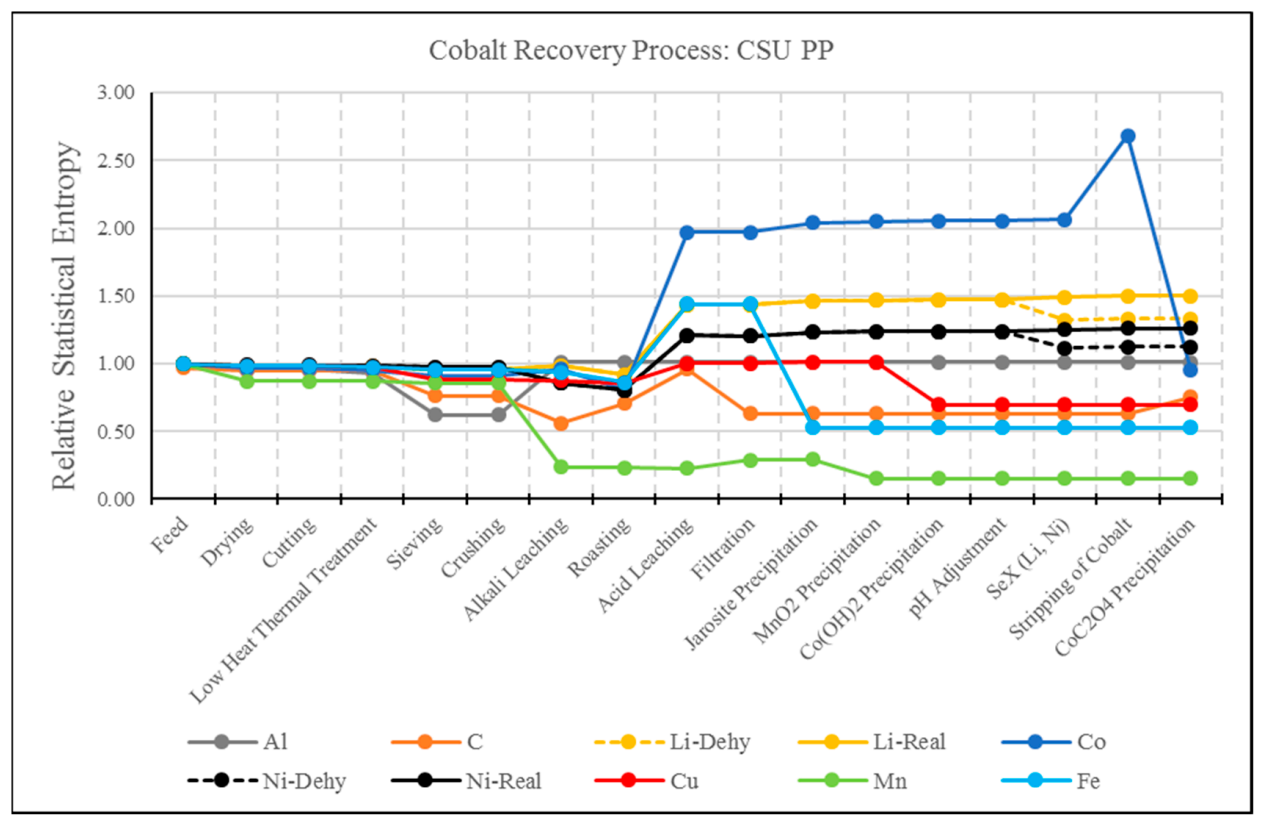

(b)

Figure 11. Electrode Treatment Loop. (a) Aalto pre-processing (PP); (b) Central South University (CSU) PP. 
Since the hydrometallurgical process reported similar recovery rates (Table 4) between the different systems studied, the difference between final elemental RSE values obtained with the Aalto and CSU PP stages (Figure 11) can be attributed to the influence of PP stages. For example, the Aalto PP presents a maximum RSE value on the Al curve of 0.5 bits while the CSU PP presents a value closer to 1.0 bits.

Furthermore, the stages of the hydrometallurgical process can be interpreted as extraction stages for different materials. From this perspective, an abrupt change in entropy should be observed when an extracted material is significantly concentrated into a single stream. In cases where the quality of the feeding material obtained by the CSU PP stages leads to undesired interactions between elements during the hydrometallurgical process, the RSE values would be negatively affected. As seen in Figure 11, the Aalto PP presents sharp changes in entropy at the extraction stage, which, from the RSE perspective, makes it a more logical choice. Admittedly, in the case of extraction as a solution, that is, change from a solid to ionic state, as it is the case of the "SeX (Li, Ni)" step, the MFA and RSE method present a drawback that requires further development. As seen, the separation of $\mathrm{Li}$ and $\mathrm{Ni}$ is not reflected in Figure 11a,b even if they are flowing into an extraction point as these elements have been effectively diluted by the leaching process. This is due to the fact that the final form of $\mathrm{Li}$ and $\mathrm{Ni}$ is heavily diluted in aqueous solution. To avoid this type of un-accuracies in future analysis, the MFA and RSE analysis can be complemented with other types of analyses, such as those applied by [19]. Nevertheless, an analysis emulating the extraction of water phase from the Li-Ni Solution (dotted Li and Ni lines in Figure 11), reflect that, indeed, there is a concentration effect of these elements during its extraction stage. Nevertheless, the final RSE value of Li and Ni suggests an overall dilution compared to its initial status. Thus, the application of MFA and RSE for the analysis of recycling processes, including solubilization, require further mathematical developments.

\section{Conclusions}

The current work presents a first attempt to use RSE and MFA analysis for the comparison of two pyrometallurgical Al-recycling processes and two hydrometallurgical treatments for the recovery of Co from LIB waste. The entropy analysis conducted lead to the following conclusions:

1. MFA and RSE methodology and analysis can be used as a tool to describe the correlation between different compounds in a process stream. As shown in this study, the addition of impurities $\left(\mathrm{Al}_{2} \mathrm{O}_{3}\right)$ has an impact on the RSE value of other compounds $(\mathrm{Al})$, reflecting an increase in entropy of Al.

2. This work demonstrates the systemic view of this methodology by identifying the correlations of entropy between elements, for example, $\mathrm{Al}$ and $\mathrm{Cu}$. In the CSU process, as $\mathrm{Al}$ is transformed to $\mathrm{Al}_{2} \mathrm{O}_{3}$ and lost to the slag phase, $\mathrm{Cu}$ is concentrated. This effect is not present in the Aalto process due to a preliminary mechanical separation step.

3. The analysis of MFA and RSE is used as a viable option for decision making as it clarifies the difference between two recycling processes and the quality of the final products is similar.

4. MFA and RSE is a methodology that weights on the influence of pre-processing stages, which were until now overlooked. After comparing RSE values, the process with the sharpest reduction in entropy in pre-processing stages also resulted in the decreased final values of RSE.

5. A drawback of the MFA and RSE for the analysis of hydrometallurgical processes has been identified. As presented, this type of analysis present biased results for elements whose extraction is based on solubilization. For example, the solvent extraction steps of $\mathrm{Li}$ and $\mathrm{Ni}$ resulted in a significant increase in entropy, even though these elements have been effectively separated from the material matrix. The use of MFA and RSE in recycling processes should be further developed to account for the effect of solubilization.

6. MFA and RSE bring a method for the analysis of streams in recycling processes that, coupled with other simulation-based analysis (e.g., exergy, and/or thermo-economics), may support the decision-making in favor of a circular economy. 
Supported by carefully designed process simulation, the positive impact of improved pre-processing stages on the final recoveries are described using MFA and RSE. This work broadens the study of resource efficiency, process simulation, MFA-RSE, and circular economy by introducing the analysis of statistical entropy to other parameters, such as those described by [19].

Author Contributions: O.V.-M. Process simulation, RSE-MFA methodology, original draft; A.P. Hydrometallurgy simulation and experimental data; K.G.v.d.B. Support on RSE analysis; A.S.-A. Conceptualization of work; M.L. Hydrometallurgy expert analysis and PI of METYK project; M.R. Rotary kiln simulation and assessment of process simulation; R.S.-G. Supervision

Funding: The research hereby presented was supported by the METYK-project (grant number 3254/31/2015), funded by Finnish innovation agency (TEKES), as well as the "Closing the loop for high-added value materials (CloseLoop, grant number 303454)" project supported by the Academy of Finland.

Acknowledgments: O.V.M. thanks the National Council of Science and Technology (CONACYT) of Mexico for a Doctoral studies scholarship. Severi Ojanen is acknowledged for his contribution to the experimental sieving of the LIB samples and Antti Roine from Outotec Oy for his help with the simulation of the Al-recycling system.

Conflicts of Interest: The authors declare no conflict of interest.

\section{References}

1. Ellen MacArthur Foundation. Towards the Circular Economy; Ellen MacArthur Foundation: Isle of Wight, UK, 2013.

2. Martin, G.; Rentsch, L.; Höck, M.; Bertau, M. Lithium market research-Global supply, future demand and price development. Energy Storage Mater. 2017, 6, 171-179. [CrossRef]

3. Linden, D.; Reddy, B.T. (Eds.) Linden's Handbook of Batteries, 4th ed.; McGraw Hill: New York, NY, USA, 2011.

4. Georgi-Maschler, T.; Friedrich, B.; Weyhe, R.; Heegn, H.; Rutz, M. Development of a recycling process for Li-ion batteries. J. Power Sources 2012, 207, 173-182. [CrossRef]

5. Heelan, J.; Gratz, E.; Zheng, Z.; Wang, Q.; Chen, M.; Apelian, D.; Wang, Y. Current and Prospective Li-Ion Battery Recycling and Recovery Processes. J. Miner. Met. Mater. Soc. 2016, 68, 2632-2638. [CrossRef]

6. Nitta, N.; Wu, F.; Lee, J.T.; Yushin, G. Li-ion battery materials: Present and future. Mater. Today 2015, 18, 252-264. [CrossRef]

7. Julien, C.M.; Mauger, A.; Zaghib, K.; Groult, H. Comparative Issues of Cathode Materials for Li-Ion Batteries. Inorganics 2014, 2, 132-154. [CrossRef]

8. Frenzel, M.; Kullik, J.; Reuter, M.A.; Gutzmer, J. Raw material “criticality"—Sense or nonsense? J. Phys. D Appl. Phys. 2017, 50, 123002. [CrossRef]

9. Chen, L.; Tang, X.; Zhang, Y.; Li, L.; Zeng, Z.; Zhang, Y. Process for the recovery of cobalt oxalate from spent lithium-ion batteries. Hydrometallurgy 2011, 108, 80-86. [CrossRef]

10. Gratz, E.; Sa, Q.; Apelian, D.; Wang, Y. A closed loop process for recycling spent lithium ion batteries. J. Power Sources 2014, 262, 255-262. [CrossRef]

11. Zheng, R.; Wang, W.; Dai, Y.; Ma, Q.; Liu, Y.; Mu, D.; Li, R.; Ren, J.; Dai, C. A closed-loop process for recycling $\mathrm{LiNixCoyMn}(1-\mathrm{x}-\mathrm{y}) \mathrm{O}_{2}$ from mixed cathode materials of lithium-ion batteries. Green Energy Environ. 2016, 2, 42-50. [CrossRef]

12. Valio, J. Critical Review on Lithium ion Battery Recycling; Aalto University: Uusimaa, Finland, 2017.

13. Zhang, T.; He, Y.; Ge, L.; Fu, R.; Zhang, X.; Huang, Y. Characteristics of wet and dry crushing methods in the recycling process of spent lithium-ion batteries. J. Power Sources 2013, 240, 766-771. [CrossRef]

14. da Costa, A.J.; Matos, J.F.; Bernardes, A.M.; Müller, I.L. Beneficiation of cobalt, copper and aluminum from wasted lithium-ion batteries by mechanical processing. Int. J. Miner. Process. 2015, 145, 77-82. [CrossRef]

15. Brunner, P.H.; Rechberger, H. Practical Handbook of Material Flow Analysis; CRC Press LLC: Boca Raton, FL, USA, 2011.

16. Bai, L.; Qiao, Q.; Li, Y.; Wan, S.; Xie, M.; Chai, F. Statistical entropy analysis of substance flows in a lead smelting process. Resour. Conserv. Recycl. 2015, 94, 118-128. [CrossRef]

17. Rechberger, H.; Graedel, T.E. The contemporary European copper cycle: Statistical entropy analysis. Ecol. Econ. 2002, 42, 59-72. [CrossRef] 
18. Martínez, O.V.; van den Boogaart, K.G.; Lundström, M.; Santasalo-Aarnio, A.; Reuter, M.; Serna-Guerrero, R. Statistical entropy analysis as tool for circular economy: Proof of concept by optimizing a lithium-ion battery waste sieving system. J. Clean. Prod. 2019, 212, 1568-1579. [CrossRef]

19. Llamas, A.A.; Delgado, A.V.; Capilla, A.V.; Cuadra, C.T.; Hultgren, M.; Peltomäki, M.; Roine, A.; Stelter, M.; Reuter, M.A. Simulation-based exergy, thermo-economic and environmental footprint analysis of primary copper production. Miner. Eng. 2019, 131, 51-65. [CrossRef]

20. Porvali, A.; Aaltonen, M.; Ojanen, S.; Velazquez-Martinez, O.; Eronen, E.; Liu, F.; Wilson, B.P.; Serna-Guerrero, R.; Lundström, M. Mechanical and hydrometallurgical processes in $\mathrm{HCl}$ media for the recycling of valuable metals from Li-ion battery waste. Resour. Conserv. Recycl. 2019, 142, 257-266. [CrossRef]

21. Dorella, G.; Mansur, M.B. A study of the separation of cobalt from spent Li-ion battery residues. J. Power Sources 2007, 170, 210-215. [CrossRef]

22. Reuter, M.A.; Hudson, C.; van Schaik, A.; Heiskanen, K.; Meskers, C.; Hagelüken, C. Metal Recycling: Opportunities, Limits, Infrastructure; United Nations Environment Programme: Nairobi, Kenya, 2013.

23. Nanoshel LCC. Aluminium Foil for Lithium Ion Battery. 2015. Available online: https://www.nanoshel.com/ product/aluminum-foil (accessed on 5 November 2018).

(C) 2019 by the authors. Licensee MDPI, Basel, Switzerland. This article is an open access article distributed under the terms and conditions of the Creative Commons Attribution (CC BY) license (http://creativecommons.org/licenses/by/4.0/). 